\title{
Efecto del microcrédito en el bienestar socioeconómico del sector transporte urbano, Chachapoyas, Amazonas
}

\section{Effect of microcredit on the socioeconomic well-being of the urban transport sector, Chachapoyas, Amazonas}

\author{
Luis Tinedo ${ }^{1}$ (iD *
}

\section{RESUMEN}

El artículo determina el efecto del microcrédito en el bienestar socioeconómico del sector transporte urbano de la ciudad de Chachapoyas, Amazonas. Para ello, se empleó los métodos inductivo - deductivo y analítico - sintético, de tipo descriptivo, además, se combinan técnicas de investigación cuantitativa, donde el instrumento de recolección de datos empleados fue el cuestionario. En tal sentido, el tipo de muestreo fue por conveniencia recopilando información de 94 transportistas. Los resultados evidencian que los ingresos familiares se incrementaron en un rango de S/ 2000 a S/ 4000, por lo tanto, las familias presentaron mejoras en las condiciones de vivienda, adquisición de activos, demanda por tecnologías digitales y formación educativa básica. En conclusión, el microcrédito tiene un efecto positivo en el bienestar socioeconómico, donde se destaca el crecimiento del ingreso familiar y la mejora de la calidad educativa de los niños y adolescentes.

Palabras clave: Ahorro, bienestar socioeconómico, transportistas.

\begin{abstract}
The article determines the effect of microcredit on the socioeconomic well-being of the urban transport sector in the city of Chachapoyas, Amazonas. For this purpose, the inductive-deductive and analyticalsynthetic methods of descriptive type were used, in addition, quantitative research techniques were combined, where the data collection instrument used was the questionnaire. In this sense, the type of sampling was by convenience, collecting information from 94 carriers. The results show that family income increased in a range of S/ 2000 to S/ 4000, therefore, the families presented improvements in housing conditions, acquisition of assets, demand for digital technologies and basic educational training. In conclusion, microcredit has a positive effect on socioeconomic wellbeing, which highlights the growth of family income and the improvement in the quality of education of children and adolescents.
\end{abstract}

Keywords: Savings, socioeconomic-well-being, transporters.

DOI: https://doi.org/10.37787/pakamuros-unj.v9i4.234

Recibido: 29/05/2021. Aceptado: 30/07/2021

* Autor para correspondencia

1. Universidad Nacional de Jaén, Perú. Email: luis tinedo@unj.edu.pe 


\section{INTRODUCCIÓN}

El microcrédito tiene un origen en el siglo XX, exactamente en 1974, gracias al aporte del economista Muhammad Yunus, de hecho, este instrumento financiero se utilizó para reducir el nivel de pobreza en Bangladesh (Mora, 2015). Existe un desafío que la sociedad debe asumir con el afán de buscar condiciones asequibles en sus miembros, no obstante, la herramienta financiera que funge como eslabón para favorecer a las familias y empresarios es el microcrédito (Alegría, 2020). Del mismo modo, esta herramienta financiera consolida una alternativa fuerte para mejorar el bienestar socioeconómico de un conglomerado, mejorar la productividad de los sectores estratégicos e incrementar la inversión local (Vallejo \& Ochoa, 2019).

Las familias de limitados recursos buscan alternativas para mejorar la calidad de vida de todos sus miembros, de hecho, el sistema financiero actual es la fuente objetiva para obtener recursos económicos y financieros. Un grupo destacado de familias relacionadas con el sector transporte emulan posibles soluciones a los inconvenientes que surgen debido a los ingresos limitados, en efecto el sector se expande en zonas urbanas y progresivamente es una alternativa para personas dependientes y de bajos recursos. Cabe destacar, que el crecimiento de la población en Latinoamérica, ha permitido la expansión de la oferta de servicios de transporte urbano en los orbes, y ello ha cambiado diferentes aspectos económicos y sociales en las ciudades (CAF, 2011).

El problema se genera en las familias que obtuvieron microcréditos pertenecientes al sector transporte, así mismo, dichos hogares cuentan con alternativas de financiamiento para lograr satisfacer sus necesidades; no obstante, el Perú, en el marco de esfuerzo nacional, permite avanzar sostenidamente hacia altos niveles de inclusión financiera, asimismo, muchas empresas del sector exploran nuevos canales y oportunidades para ofrecer y acceder a servicios financieros, en tal sentido, el microcrédito en los últimos años se volvió una herramienta fundamental para luchar contra la disparidad de los ingresos (García, 2021).

La inclusión financiera y el acceso a diferentes créditos, hicieron que el sistema financiero creciera de forma considerable, sin embargo, existen algunos vacíos en referencia a los estudios de impacto y efectos reales que puedan ocasionar los microcréditos en el bienestar y calidad de vida de las familias de algún sector estratégico, como lo es el transporte. De hecho, Prieto (2018), denota el valor de los servicios microfinancieros en las personas naturales que difícilmente son sujetos a créditos, también enmarca el impacto de la amplitud de las instituciones microfinancieras en la actividad económica regional y por ende en las zonas catalogadas pobres del Perú, vinculado a ello, Aguilar et al. (2017), sostiene claramente 
que los microcréditos forman un eslabón prioritario para generar microempresas que impulsan la empleabilidad; así mismo, resalta que el incremento de las unidades monetarias en los jefes o jefas de hogar mejora el bienestar familiar, de igual forma, los programas de gobierno social ayudan a que el 77.3\% de las familias utilicen dicho crédito para fomentar la creación de una microempresa, cabe resaltar, que el dinero también lo usan para reducir la brecha de los ingresos. Manrique et al. (2017), desarrollo un modelo de impacto donde destaca que los receptores de los servicios microcrediticos aumentan sus activos en $17.89 \%$ con el objetivo de acrecentar los sistemas productivos rurales. Assis et al. (2021), resalta el efecto de los programas microcrediticos rurales en las actividades agrícolas, pues, el indicador costo beneficio repercute en el incremento moderado del retorno con respecto a la inversión, ligado a ello, los efectos del programa en los beneficiarios representan el 3.5\% de la producción agrícola rural, por cual el 22\% de la producción es ganadera. La imagen del microcrédito a una tasa factible con periodos de pago asequibles y montos provechosos dinamiza la economía, generando así en las familias un incremento significativo de activos y potencializar las microempresas familiares (Maguiña et al., 2021). Rivera \& Cardozo (2019), descubrieron que esta herramienta crediticia no solo mejora las actividades comerciales, sino también resalta una gran mejoría en la calidad de vida familiar, trabajo y vivienda, cabe mencionar que los microcréditos se utilizan para fomentar el desarrollo económico local. También es preciso resaltar que Alegre \& Alarcón (2016) añaden elementos de impacto en las familias de transportistas ocasionado por el microcrédito, para bien, en el sector transporte existe una gran demanda por servicios de traslados terrestres en distintas zonas de una ciudad generando ingresos en términos monetarios. Los impactos microcrediticios influyen en los ámbitos empresarial y hogar (Gavilanes y Romero, 2015), resaltando, un holgado nivel de ingresos domésticos conjugados a nuevos emprendimientos empresariales (Ayuque, 2014).

En síntesis, se pretende determinar el efecto del microcrédito en el bienestar socioeconómico del sector transporte urbano en la ciudad de Chachapoyas, Amazonas, resaltando el hecho que las empresas ligadas al sistema financiero buscan alternativas de nichos de mercado poco explorado con el objetivo de lograr cobertura microcrediticia en la mayor parte de hogares de la región.

\section{MATERIALES Y MÉTODOS}

Se desarrolló bajo un enfoque cuantitativo, ejecutándose un diseño no experimental con el propósito de recabar información relevante de las variables en cuestión, por esta razón, los métodos utilizados se justifican en el análisis inductivo - deductivo y analítico - sintético. Tiene un tipo de muestreo no 
probabilístico por conveniencia, debido a que la muestra solo representa a 94 transportistas ubicados en el perímetro urbano de la ciudad de Chachapoyas.

La información recaudada se estructuró bajo la percepción de la situación económica y social de las personas encuestadas, es por ello no se solicitaron montos específicos de gastos e ingresos debido a que muchos de ellos no cuentan con esta información, por lo tanto, para facilitar el muestreo se tomó como referencia los datos de los transportistas que solo recibieron microcrédito. El instrumento para recaudar la información fue el cuestionario, no obstante, se utilizó el coeficiente Alfa Cronbach, evidenciando un excelente nivel de confiabilidad (Tabla 1). El programa utilizado fue el SPSS versión 20, de esta forma se analizaron los datos aplicando las herramientas de la estadística descriptiva a través del uso de tablas de frecuencias.

Tabla 1. Estadísticos de fiabilidad

\begin{tabular}{ccc}
\hline $\begin{array}{c}\text { Alfa de } \\
\text { Cronbach }\end{array}$ & $\begin{array}{c}\text { Alfa de Cronbach basada en elementos } \\
\text { estandarizados }\end{array}$ & $\begin{array}{c}\text { N de } \\
\text { elementos }\end{array}$ \\
\hline 0.818 & 0.826 & 13 \\
\hline
\end{tabular}

Los efectos en el bienestar socioeconómico de las familias de los transportistas que percibieron el microcrédito se describen en dos áreas prioritarias: hogar y educación. Con respecto al hogar, los análisis se realizaron a partir de los cambios en ingresos, ahorros, vivienda, adquisición de activos y servicios básicos. Así también, en el ámbito educativo, se describe la evolución del nivel formativo e incremento actividades extracurriculares de los hijos en edad escolar.

\section{RESULTADOS}

En la Tabla 2, se muestra los resultados del ingreso mensual de los transportistas antes y después del microcrédito, donde el rango máximo de dinero obtenido oscila entre S/ 1000 y S/ 4000. El 81.9\% de los encuestados antes de recibir el crédito microfinanciero percibió un ingreso máximo de S/ 1000, además, el 70.2\% y 23.4\% de los hogares percibieron un ingreso entre los intervalos de S/ 2000 a S/ 3000 y S/ 3000 a S/ 4000 respectivamente.

Tabla 2. Rango de ingreso mensual de los transportistas antes y después del microcrédito

\begin{tabular}{ccc}
\hline Rango en soles & Porcentaje antes del microcrédito & $\begin{array}{c}\text { Porcentaje después del } \\
\text { microcrédito }\end{array}$ \\
\hline Entre $0-1000$ & 81,9 & 0 \\
Entre $1000-2000$ & 18.1 & 6,4 \\
Entre $2000-3000$ & 0 & 70,2 \\
Entre $3000-4000$ & 0 & 23,4 \\
\hline
\end{tabular}


La Tabla 3, se muestra la percepción de la capacidad de ahorro familiar, donde el $71.3 \%$ de los encuestados determinan que existe una fuerte capacidad de ahorro luego de percibir el microcrédito. Además, un 24.5\% manifestaron que existió un incremento moderado y el $4.3 \%$ determinó que la capacidad de ahorro familiar no aumento.

Tabla 3. Capacidad de ahorro familiar después del microcrédito

\begin{tabular}{lcccc}
\hline \multicolumn{1}{c}{ Escala } & Frecuencia & Porcentaje & Porcentaje válido & $\begin{array}{c}\text { Porcentaje } \\
\text { acumulado }\end{array}$ \\
\hline Mucho & 67 & 71.3 & 71.3 & 71.3 \\
Más o menos & 23 & 24.5 & 24.5 & 95.7 \\
No ha aumentado & 4 & 4.3 & 4.3 & 100.0 \\
Total & 94 & 100.0 & 100.0 & \\
\hline
\end{tabular}

En termino de adquisición o alquiler de vivienda se evidencia un cambio sustancial en las condiciones y mejoramiento estructural del hogar. La Tabla 4 indica la efectividad del microcrédito en la vivienda, debido a que el $80.9 \%$ de los encuestados manifestaron que adquirieron o alquilaron una vivienda.

Tabla 4. Transportistas encuestados según la adquisición o alquiler de vivienda

\begin{tabular}{lcccc}
\hline Escala & Frecuencia & Porcentaje & Porcentaje válido & $\begin{array}{c}\text { Porcentaje } \\
\text { acumulado }\end{array}$ \\
\hline Si & 76 & 80.9 & 80.9 & 80.9 \\
No & 18 & 19.1 & 19.1 & 100.0 \\
Total & 94 & 100.0 & 100.0 & \\
\hline
\end{tabular}

La Tabla 5 contextualiza la relación entre los ingresos económicos y las condiciones de la vivienda después del microcrédito, donde el 33.0\% y 18.1\% de los encuestados expresaron una mejoría en condiciones del hogar, debido el incremento del ingreso expresado en los intervalos S/ 2000 a S/ 3000 y S/ 3000 a S/ 4000 respectivamente; de hecho, también existe evidencia que el 24.5\% de los transportistas no mostraron mejorías en las condiciones de vivienda. 
Tabla 5. Relación del ingreso económico y condiciones de vivienda después del microcrédito

\begin{tabular}{|c|c|c|c|c|c|c|}
\hline \multicolumn{3}{|c|}{ Rango en soles } & \multirow{2}{*}{$\begin{array}{c}\begin{array}{c}\text { Mejores } \\
\text { condiciones }\end{array} \\
4\end{array}$} & \multirow{2}{*}{$\begin{array}{c}\begin{array}{c}\text { Iguales } \\
\text { condiciones }\end{array} \\
0\end{array}$} & \multirow{2}{*}{$\begin{array}{c}\begin{array}{c}\text { No alquilo o } \\
\text { compro } \\
\text { vivienda }\end{array} \\
2\end{array}$} & \multirow{2}{*}{$\begin{array}{c}\text { Total } \\
6\end{array}$} \\
\hline \multirow{6}{*}{$\begin{array}{c}\text { Nivel de } \\
\text { ingreso } \\
\text { después del } \\
\text { microcrédito }\end{array}$} & \multirow{2}{*}{$1000-2000$} & Recuento & & & & \\
\hline & & $\%$ del total & $4.3 \%$ & $0.0 \%$ & $2.1 \%$ & $6.4 \%$ \\
\hline & \multirow{2}{*}{$2000-3000$} & Recuento & 31 & 20 & 15 & 66 \\
\hline & & $\%$ del total & $33.0 \%$ & $21.3 \%$ & $16.0 \%$ & $70.2 \%$ \\
\hline & \multirow{2}{*}{$3000-4000$} & Recuento & 17 & 3 & 2 & 22 \\
\hline & & $\%$ del total & $18.1 \%$ & $3.2 \%$ & $2.1 \%$ & $23.4 \%$ \\
\hline \multirow{2}{*}{\multicolumn{2}{|c|}{ Total }} & Recuento & 52 & 23 & 19 & 94 \\
\hline & & $\%$ del total & $55.3 \%$ & $24.5 \%$ & $20.2 \%$ & $100.0 \%$ \\
\hline
\end{tabular}

La Tabla 6 muestra que el 24.5\% de los encuestados efectuó una remodelación o construcción de alguna área de la vivienda, así mismo, el 33.0\% hizo una remodelación con adquisición de algunos artículos necesarios para la vivienda.

Tabla 6. Tipo de remodelación de vivienda

\begin{tabular}{lcccc}
\hline \multicolumn{1}{c}{ Alternativa } & Frecuencia & Porcentaje & $\begin{array}{c}\text { Porcentaje } \\
\text { válido }\end{array}$ & $\begin{array}{c}\text { Porcentaje } \\
\text { acumulado }\end{array}$ \\
\hline Remodelación o construcción de alguna área de & 23 & 24.5 & 24.5 & 24.5 \\
la vivienda & 31 & 33.0 & 33.0 & 57.4 \\
Remodelación de hogar con compra de artículos & 22 & 23.4 & 23.4 & 80.9 \\
Ambos & 18 & 19.1 & 19.1 & 100.0 \\
Ninguno & 94 & 100.0 & 100.0 & \\
Total & & & & \\
\hline
\end{tabular}

Luego de obtener el microcrédito los transportistas adquirieron diversos artefactos. En particular, la Tabla 7 visualiza que el $42.6 \%$ de los transportistas adquirió artefactos de línea blanca (específicamente cocina, lavadora y secadora), y el 10.6\% adquirió artefactos de línea marrón (específicamente televisor y equipo de sonido).

Tabla 7. Artefactos adquiridos después del microcrédito

\begin{tabular}{lcccc}
\hline \multicolumn{1}{c}{ Alternativa } & Frecuencia & Porcentaje & Porcentaje válido & $\begin{array}{c}\text { Porcentaje } \\
\text { acumulado (\%) }\end{array}$ \\
\hline Línea blanca & 40 & 42.6 & 42.6 & 42.6 \\
Línea marrón & 10 & 10.6 & 10.6 & 53.2 \\
Ambos & 28 & 29.8 & 29.8 & 83.0 \\
Ninguno & 16 & 17.0 & 17.0 & 100.0 \\
Total & 94 & 100.0 & 100.0 & \\
\hline
\end{tabular}


En la Tabla 8 existe evidencia, que las familias adquirieron tecnologías digitales, como computadoras (41.5\%), televisión por cable (36.2\%) y teléfonos móviles (14.9\%).

Tabla 8. Tecnologías adquiridas después del microcrédito

\begin{tabular}{lcccc}
\hline \multicolumn{1}{c}{ Alternativa } & Frecuencia & Porcentaje & Porcentaje válido & $\begin{array}{c}\text { Porcentaje } \\
\text { acumulado }\end{array}$ \\
\hline Computadora & 39 & 41.5 & 41.5 & 41.5 \\
Internet & 2 & 2.1 & 2.1 & 43.6 \\
Televisión por cable & 34 & 36.2 & 36.2 & 79.8 \\
Teléfono móvil & 14 & 14.9 & 14.9 & 94.7 \\
inteligente & 5 & 5.3 & 5.3 & 100.0 \\
Ninguno & 94 & 100.0 & 100.0 & \\
Total & & & & \\
\hline
\end{tabular}

La Tabla 9 determina que el 12.8\% de los encuestados adquirieron un vehículo de transporte y el $87.2 \%$ no lo hizo, después de percibir un beneficio microcrediticio.

Tabla 9. Vehículos adquiridos

\begin{tabular}{lcccc}
\hline \multicolumn{1}{c}{ Alternativa } & Frecuencia & Porcentaje & Porcentaje válido & $\begin{array}{c}\text { Porcentaje } \\
\text { acumulado }\end{array}$ \\
\hline Un (1) vehículo & 12 & 12.8 & 12.8 & 12.8 \\
Ninguno & 82 & 87.2 & 87.2 & 100.0 \\
Total & 94 & 100.0 & 100.0 & \\
\hline
\end{tabular}

La Tabla 10 muestra que el 100\% de los transportistas encuestados tienen una percepción de mejora en los servicios básicos luego de obtener el microcrédito.

Tabla 10. Percepción de mejora en servicios básicos

\begin{tabular}{ccccc}
\hline Percepción & Frecuencia & Porcentaje & Porcentaje válido & $\begin{array}{c}\text { Porcentaje } \\
\text { acumulado }\end{array}$ \\
\hline $\mathrm{Si}$ & 94 & 100.0 & 100.0 & 100.0 \\
\hline
\end{tabular}

\section{Efectos en el nivel educativo}

La Tabla 11 evidencia que el $77.7 \%$ de los transportistas solo tienen un hijo en edad escolar, un $18.1 \%$ tiene dos hijos en edad escolar y el 4.3\% no tienen ningún hijo en edad escolar. 
Tabla 11. Hijos en edad escolar

\begin{tabular}{lcccc}
\hline \multicolumn{1}{c}{ Alternativa } & Frecuencia & Porcentaje & Porcentaje válido & $\begin{array}{c}\text { Porcentaje } \\
\text { acumulado }\end{array}$ \\
\hline Un (1) hijo & 73 & 77.7 & 77.7 & 77.7 \\
Dos (2) hijos & 17 & 18.1 & 18.1 & 95.7 \\
No tiene hijos en & 4 & 4.3 & 4.3 & 100.0 \\
edad escolar & 94 & 100.0 & 100.0 & \\
Total & 9 & & \\
\hline
\end{tabular}

De acuerdo a la Tabla 12, el 95.7\% de los hijos de transportistas asisten a la escuela pública o privada, así mismo, el resto de los encuestados (4.3\%) determino que no tenía hijos en edad escolar.

Tabla 12. Hijos que asisten a la escuela

\begin{tabular}{lcccc}
\hline \multicolumn{1}{c}{ Alternativa } & Frecuencia & Porcentaje & Porcentaje válido & $\begin{array}{c}\text { Porcentaje } \\
\text { acumulado }\end{array}$ \\
\hline Todos & 90 & 95.7 & 95.7 & 95.7 \\
Ninguno & 4 & 4.3 & 4.3 & 100.0 \\
Total & 94 & 100.0 & 100.0 & \\
\hline
\end{tabular}

La Tabla 13, denota que los hijos de los transportistas gozaron de una mejor calidad educativa (77.7\%) además, el 18.1\% manifestó que la educación de los niños y jóvenes mejoró de manera no significativa.

Tabla 13. Mejora de la calidad educativa de los hijos de transportistas

\begin{tabular}{lcccc}
\hline \multicolumn{1}{c}{ Alternativa } & Frecuencia & Porcentaje & Porcentaje válido & $\begin{array}{c}\text { Porcentaje } \\
\text { acumulado }\end{array}$ \\
\hline Alto & 73 & 77.7 & 77.7 & 77.7 \\
Medio & 17 & 18.1 & 18.1 & 95.7 \\
No ha mejorado & 4 & 4.3 & 4.3 & 100.0 \\
Total & 94 & 100.0 & 100.0 & \\
\hline
\end{tabular}

La Tabla 14 determina que el 91.5\% de los transportistas matriculó a los hijos en actividades extracurriculares. Estos resultados establecen que el microcrédito es importante y ejemplifica una superlativa calidad educativa, ligado a la utilización de actividades extracurriculares con el fin de que los niños y adolescentes gocen de mejores oportunidades.

Tabla 14. Actividades extracurriculares de los hijos de transportistas

\begin{tabular}{lcccc}
\hline Alternativa & Frecuencia & Porcentaje & Porcentaje válido & $\begin{array}{c}\text { Porcentaje } \\
\text { acumulado }\end{array}$ \\
\hline $\mathrm{Si}$ & 86 & 91.5 & 91.5 & 91.5 \\
No & 8 & 8.5 & 8.5 & 100.0 \\
Total & 94 & 100.0 & 100.0 & \\
\hline
\end{tabular}




\section{DISCUSIÓN}

Los resultados proporcionados por la Tabla 3, muestran que los microcréditos son efectivos para lograr aumentar los ingresos, es decir, los transportistas luego del beneficio financiero obtuvieron rentas monetarias entre S/ 2000 - S/ 4000; Aguilar et al. (2017) y Prieto (2018), las familias usan dicho beneficio para obtener condiciones favorables, principalmente, reducir brechas de ingreso, además, bajo la perspectiva de García (2021) y Mora (2015), los niveles de inclusión financiera en los sectores menos favorecidos proporcionan una oportunidad para aumentar los ingresos económicos de las familias y consecuentemente reducir la pobreza.

Con relación a los activos demandados, las familias adquirieron artefactos de línea blanca y marrón (29.9\%), equipos y servicios tecnológicos (94.7\%), y vehículos de transporte (12.8\%); que según Manrique et al. (2017) y Maguiña et al. (2021) bajo modelos de impacto determinaron que el aumento de activos es recurrente en las familias sujetos a microcréditos, de hecho, (Rivera, 2012) coincide en que los créditos pequeños aumentan la adquisición de activos.

Con respecto a la Tabla 9 el 12.8\% de los encuestados compraron un vehículo para brindar el servicio de transporte, donde según Mantilla (2012), dicha adquisición es aceptable porque fomenta la competencia en el sector. No obstante, el sector transporte como eje de la economía local no es único beneficiado por el microcrédito, también según Assis et al. (2021), Cordobés \& Iglesias (2011) y Rivera \& Cardozo (2019) los programas microcrediticos incentivan el empleo y desarrollo local a través de iniciativas empresariales agrícolas y ganaderas.

Los microcréditos incrementan el nivel educativo de los hijos de transportistas (77.7\%), ello, debido a la adquisición de tecnologías digitales y mayor acceso a la información; así mismo, el 91.5\% de los jefes de familia transportistas matricularon a sus hijos en actividades extracurriculares, construyendo en los niños mejores oportunidades para el futuro, de hecho, según CAF (2011) y Vallejo \& Ochoa (2019), los microcréditos surgen como incentivo para mejorar el bienestar socioeconómico, aumentar la oferta de servicios de transporte y mejorar la inversión local en educación regular.

Finalmente, existe evidencia sobre el incremento de las condiciones de vivienda (33.0\%) y remodelación (24.5\%) dado el incremento de los ingresos mensuales, de hecho, la situación de los hogares también fue favorable, por lo tanto, según Gavilanes \& Romero (2015), Ayuque (2014) y Mantilla (2012) el ingreso financiero permite realizar emprendimientos empresariales, mejorar la estructura de vivienda e impulsar ámbitos de confort familiar. En definitiva, el sector transporte urbano en la ciudad de Chachapoyas está 
fortaleciendo la cultura financiera, porque este segmento es importante para el crecimiento y bienestar socioeconómico de las familias.

\section{CONCLUSIONES}

El microcrédito ha generado un efecto positivo en el hogar, especialmente, los ingresos de los transportistas se incrementaron entre S/ 2000 a S/ 4000 soles, de igual forma, existe una fuerte capacidad de ahorro, mejores condiciones de vivienda, remodelación del hogar, adquisición de artefactos de línea blanca (cocina, lavadora y secadora) y marrón (televisor y equipo de sonido), compra de equipos electrónicos (computadora y teléfono móvil), adquisición de vehículo de transporte y mejora de los servicios básicos. No obstante, el beneficio microcrediticio también genera un efecto positivo calidad educativa de los niños, asimismo, los jefes del hogar matricularon a sus hijos en actividades extracurriculares con el afán de consolidar el nivel de calidad educativa.

Consecuentemente, se resalta la importancia que tiene el microcrédito en el sector transporte ya que permite acrecentar las condiciones de vida de sus miembros frente a las posibilidades del progreso regional.

\section{REFERENCIAS BIBLIOGRÁFICAS}

Aguilar Prieto, E., Uñón Pablos, E., \& Morales Barragán, F. (2017). Microcrédito y pobreza. La experiencia del programa Microempresas Sociales de Banmujer en Chiapas. Economía, sociedad y territorio, XVII(55), 809-835. doi: http://dx.doi.org/10.22136/est2017885

Alegre Escorza, M., \& Alarcón Rodriguez-Paiva, G. (2016). Transporte Urbano:¿Como resolver la movilidad en Lima y Callao? Lima: CIES.

Alegría, G. (21 de Abril de 2020). Incremento de los Microcréditos en el Perú. Centrum Think. https://centrumthink.pucp.edu.pe/publicaciones/incremento-de-los-microcreditos-en-el-peru/

Assis Guedes, I., Cavalcanti Almeida, A., y Oliveira de Siqueira, L. B. (2021). Efectos del microcrédito rural en la producción agrícola de la región Nordeste: evidencia del Programa Agroamigo. Revista de Economía y Sociología Rural, 59(1), 1-19. doi: https://doi.org/10.1590/1806$\underline{9479.2021 .210774}$

Ayuque Huamán, L. L. (2014). Los microcréditos en el crecimiento económico de los pequeños empresarios del Distrito de Huancavelica año 2012 [ Tesis de título, Universidad Nacional de Huancavelica]. https://repositorio.unh.edu.pe/handle/UNH/321 
CAF. (2011). Desarrollo urbano y movilidad en América Latina. Editorial del Banco de Desarrollo de América Latina.

https://scioteca.caf.com/bitstream/handle/123456789/419/omu.pdf?sequence=1\&isAllowed=y

García Villacorta, A. (23 de Junio de 2021). ¿Por qué urge impulsar la inclusión financiera en el Perú?.

Conexión Esan.

https://www.esan.edu.pe/conexion/actualidad/2021/06/23/por-que-urge-impulsar-la-inclusionfinanciera-en-el-peru/

Gavilanes Rivadeneira, A. V., \& Romero Tintín, M. E. (2015). Medición del impacto de los microcréditos sobre el nivel de vida de los habitantes del Cantón San Fernando, año 2014 [Tesis de Título, Universidad de Cuenca]. http://dspace.ucuenca.edu.ec/handle/123456789/22306

Maguiña Palma, M. E., Ramírez Asís, E. H., Huerta Soto, R. M., \& Concepción Lázaro, R. J. (2021). Microcrédito y desarrollo de las microempresas en las zonas rurales de Ancash, Perú. Ciencia ergo - sum, 28(1), 1-8. doi: https://doi.org/10.30878/ces.v28n1a3

Manrique Joya, G., Ramírez, M., \& Santos Varó, F. (2017). Impacto del microcrédito sobre la pobreza rural en los municipios de Tunja y Samacá, Colombia. Semestre Económico, 20(45), 51-76 . doi: https://doi.org/10.22395/seec.v20n45a2

Mora Casal, R. (2015). La empresa social de Muhammad Yunus, un nuevo paradigma para erradicar la pobreza. Economía \& Sociedad, 20(47), 1-18. doi: http://dx.doi.org/10.15359/eys.20-47.3

Prieto Martínez, J. (9 de Enero de 2018). La Participación De Las Microfinanzas en el Desarrollo Económico. Instituto del Sur. https://www.isur.edu.pe/es/articulo/escuela-de-negocios/laparticipacion-de-las-microfinanzas-en-el-desarrollo-economico

Rivera Perales, A. M., \& Cardozo Romero, J. R. (2019). Impacto del microcrédito en el mercado. Fides et Ratio - Revista de Difusión cultural y científica de la Universidad La Salle en Bolivia, 18(18), 177-22. http://www.scielo.org.bo/pdf/rfer/v18n18/v18n18_a10.pdf

Vallejo Ramírez, J. B., \& Ochoa Herrera, M. J. (2019). Evolución del microcrédito del sector popular y solidario frente a la banca pública - privada del Ecuador. Revista ECA Sinergia, 10(2), 138-148. https://dialnet.unirioja.es/servlet/articulo?codigo=6965734

(c) (1) Copyright $\mathbb{C}$ de los autores. Titular de la licencia: Revista Pakamuros. Este artículo es un artículo de acceso abierto distribuido bajo los términos y condiciones de la licencia Creative Commons (CC BY-NC) (https://creativecommons.org/licenses/by-nc/4.0/ ). 